\title{
Avionics Architectures for Exploration: Building a Better Approach for (Human) Spaceflight Avionics
}

\author{
Montgomery B. Goforth ${ }^{1}$, James E. Ratliff ${ }^{2}$, Kevin L. Hames ${ }^{3}$, and Sharada V. Vitalpur ${ }^{4}$ \\ NASA Johnson Space Center, Houston, Texas, 77058
}

The field of Avionics is advancing far more rapidly in terrestrial applications than in space flight applications. Spaceflight Avionics are not keeping pace with expectations set by terrestrial experience, nor are they keeping pace with the need for increasingly complex automation and crew interfaces as we move beyond Low Earth Orbit. NASA must take advantage of the strides being made by both space-related and terrestrial industries to drive our development and sustaining costs down. This paper describes ongoing efforts by the Avionics Architectures for Exploration (AAE) project chartered by NASA's Advanced Exploration Systems (AES) Program to evaluate new avionic architectures and technologies, provide objective comparisons of them, and mature selected technologies for flight and for use by other AES projects. Results from the AAE project's FY13 efforts are discussed, along with the status of FY14 efforts and future plans.

\section{Introduction}

The field of Avionics is advancing far more rapidly in terrestrial applications than in space flight applications. Spaceflight Avionics are not keeping pace with expectations set by terrestrial experience, nor are they keeping pace with the need for increasingly complex automation and crew interfaces as we move beyond Low Earth Orbit. NASA must take advantage of the strides being made by both space-related and terrestrial industries to drive our development and sustaining costs down. This paper describes ongoing efforts by the Avionics Architectures for Exploration (AAE) project chartered by NASA's Advanced Exploration Systems (AES) Program to evaluate new avionic architectures and technologies, provide objective comparisons of them, and mature selected technologies for flight and for use by other AES projects. The AAE project team includes members from most NASA centers, and from industry.

It is our intent to develop a common core avionic system that has standard capabilities and interfaces, and contains the basic elements and functionality needed for any spacecraft. This common core will be scalable and tailored to specific missions. It will incorporate hardware and software from multiple vendors, and be upgradeable in order to infuse incremental capabilities and new technologies. It will maximize the use of reconfigurable open source software (e.g., Goddard Space Flight Center's (GSFC’s) Core Flight Software (CFS)).

Our long-term focus is on improving functionality, reliability, and autonomy, while reducing size, weight, and power. Where possible, we will leverage terrestrial commercial capabilities to drive down development and sustaining costs. We will select promising technologies for evaluation, compare them in an objective manner, and mature them to be available for future programs.

Our first major goal for FY13 was to demonstrate a plausible avionics architecture for a notional space station located at the $2^{\text {nd }}$ Earth-Moon Lagrange point (hereafter referred to as an "L2 Station"). This architecture was meant to provide a reasonable set of capabilities using avionics hardware that was either flight ready, or that could be made flight ready within 2 to 3 years.

Our second major goal for FY13 was to provide a flexible avionics architecture that can be used to evaluate future concepts/architectures/components for both an L2 Station and other vehicles. This flexible architecture

\footnotetext{
${ }^{1}$ Strategic Planning and Partnerships, Avionic Systems Division, 2101 NASA Parkway/EV1

${ }^{2}$ Associate Division Chief, Avionic Systems Division, 2101 NASA Parkway/EV1

${ }^{3}$ Technology Systems Integration, Avionic Systems Division, 2101 NASA Parkway/EV7

${ }^{4}$ Chief Communications Architect, Avionic Systems Division, 2101 NASA Parkway/EV8
} 
accommodates equipment from multiple vendors in order to benchmark performance against a specific mission. It should allow us to take advantage of advances in technology being made by industry to drive our development and sustaining costs down, and facilitate efforts to incrementally infuse and validate architectural components in the future.

The remainder of this paper describes our approach, technical areas of emphasis, integrated test experience and results, and future plans. As a part of the AES Program, we are encouraged to set aggressive goals and fall short if necessary, rather than to set our sights too low. We are also asked to emphasize providing our personnel with handson experience in development, integration, and testing. That we have embraced both of these philosophies will be evident in the descriptions below.

\section{Approach}

Our overall approach emphasizes the need for testing of different alternatives to provide objective evaluations of the relative merits of architectures and technologies. Technologies selected for evaluation include both "legacy" systems (e.g., MIL-STD-1553B), and those included in technology roadmaps produced by NASA's Office of Chief Technologist (OCT), Avionics Steering Committee (ASC), and Space Communications and Navigaton (SCaN) Office. We recognize that any future exploration vehicles will likely be composed of a cluster of more specialized vehicles deployed at different times by various organizations/contractors (perhaps from different countries), and that we must address how these specialized vehicles interact during all mission phases. Although we are focused on avionics for Human Spaceflight, we are considering technologies applicable for both crewed and robotic vehicles.

Operations on both the Space Shuttle and International Space Station (ISS) have shown the importance of an Ethernet LAN on a vehicle for crew use, both to ship large volumes of data (including imagery) and to enable the use of terrestrially available Commercial Off-The-Shelf (COTS) products (hardware and software). Both of these vehicles were initially designed without an Ethernet Local Area Network (LAN) which was then added at significant cost. The Space Shuttle was designed before the wide availability of LAN technology of any kind. The ISS was designed with a Payload Ethernet Hub Gateway (PEHG), but this capability was limited to routing payload data, principally for downlink via the Ku-Band system; it did not provide anything like a LAN for the crew to use.

Based on our experience, Ethernet will be needed for any future crewed mission, and we want to leverage it for both COTS products and our terrestrial experience base. Hence, we decided to treat Ethernet as a fundamental part of the onboard architecture, and then evaluate what capabilities need to be added for command and control functions. Our initial areas of investigation center on the use of time-triggered and non-deterministic Ethernet for all vehicle functions. We are also looking at ways to interface an Ethernet backbone with other control bus protocols (1553B, SpaceWire, etc.).

In addition, we are emphasizing the investigation of human interfaces; more powerful processors and network configurations; wireless technologies for both networking and instrumentation; and flexible long-haul communications technology.

\section{A.High-Level Challenges and Guidelines}

As part of our initial efforts, we identified a set of high level challenges:

- Future exploration vehicles are undefined, but are likely to be an aggregate of multiple vehicles from multiple sources. This will drive sparing, redundancy, etc.

- Size, Weight, and Power (SWAP) must always be minimized

- Processing requirements exceed that which can be provided by existing Space Hardened Avionics (e.g., Power PC-based Rad750)

- The Radiation Environment at HEO and beyond is much worse than it is at LEO (the environment with which HSF has the most operational experience).

- Because of the radiation environment, we cannot rely on COTS hardware for additional processing capabilities as we have done on Shuttle and ISS (i.e., laptops aren't likely to work reliably)

- Exploration vehicle requirements will change/grow over the vehicle's lifetime, as will the expectations set by Terrestrial State-of-the-Art. We need to accommodate these changes without undue expense. 
These high level challenges, along with other project level decisions, lead to the following set of architectural guidelines:

- Minimize Avionics SWAP in the Flight Vehicle. Use wireless LANs and sensor networks where possible. Use low/no power sensors ("Zero Wire”) whenever practical. Minimize total wiring on the vehicle (using topologies, VPNs, etc.). Minimize unique components for sparing.

- Keep the architecture and design modular so we can launch incrementally. Allow capabilities to be integrated into the vehicle when they are needed, or earlier if it makes sense from an available launch mass perspective.

- Minimize Cost. Use existing capabilities to avoid near-term DDT\&E. Allow for growth using new technology to avoid future DDT\&E. Allow for infusion of new technology to reduce sustaining effort. Look for places where improved Avionics can drive down overall vehicle costs.

- Minimize Risk. Use proven technology for critical functions. Use existing capabilities to minimize schedule risk.

- Minimize logistics and maintenance. Pay particular attention to the trade-off between utilizing precious habitable volume for mounting avionics inside the spacecraft versus the effort required for external maintenance via EVA.

- Support Heterogeneity. We cannot expect every module of an aggregate vehicle to be the same. No one architecture/design will be an acceptable answer for everything.

- Strive for "Commonality". This cannot mean picking a set of components/boards/boxes to be used in multiple vehicles developed over long periods of time by different vendors, but it could mean picking the same components/boards/boxes to be used throughout a vehicle. It must mean developing a way for these different things to talk to each other. It should mean making sure that things of a similar type can be exchanged, to allow for minimal sparing.

- Provide Ethernet on the vehicle for crew support.

- Maximize the use of Core Flight Software (CFS). Another AES-funded effort at JSC is the Core Flight Software (CFS) project. Briefly stated, the Core Flight Software Project's objective is to evolve and extend the reusability of GSFC's Core Flight Software System into human-rated systems, thus enabling low cost, and rapid access to space. It was decided that the AAE project would make maximum use of CFS. This approach should maximize our ability to leverage platforms, resources and skills from synergetic programs/projects for development of next generation human-rated space software systems and utilize these products in direct support of development and certification of future manned programs.

- Use IPAS and F.F. In order to maximize our return on investment, the AAE project has made extensive use of the Flight Deck of the Future (F.F) and the Integrated Power, Avionics, and Software (IPAS) capability developed by JSC Engineering. IPAS is multi-system environment where next generation flight systems can be tested and demonstrated. It provides multi-mission/multi-vehicle simulations, a common set of test services, access to a variety of actual sensors and effectors, and access via the Distributed Simulation Network (DSNet) to capabilities at multiple NASA centers ${ }^{1}$. The F.F focuses primarily on the humanmachine interface. It allows us to evaluate different interface technologies together with personnel from Flight Crew Operations, Human Health and Performance, and other stakeholders.

\section{B. Standards and Specifications}

We established a lean set of interface standards \& specifications for our use in FY13, with the expectation that they would be refined and extended as needed for our future efforts.

Table 1. Interface Standards and Specifications

\begin{tabular}{|l|l|}
\hline Interface Type & Standard/Specification \\
\hline 1553 & MIL-STD-1553B \\
\hline Space Wire & ECSS E-ST-50-12C \\
\hline Low Speed Serial & EIA Standard RS-232-C \\
\hline High Speed Serial & EIA Standard RS-422 \\
\hline Ethernet & IEEE $802.3^{\mathrm{TM}}-2005$ \\
\hline Time Triggered Ethernet (TTE) & SAE AS6802-2011 \\
\hline Wireless LAN & IEEE $802.11^{\mathrm{TM}}-2005$ \\
\hline Wireless Sensors & ISA 100.11a-2012 \\
\hline & 3 \\
\hline
\end{tabular}

American Institute of Aeronautics and Astronautics 


\section{Requirements}

Generic, high-level avionic system requirements were developed to aid in the objective evaluation of different architectures. Like the standards \& specifications, these were established with the expectation that they would be refined and extended as needed for our future efforts. These requirements were not formally derived, but were established through a process of brainstorming, comparison with mission requirements sets being developed by NASA in the same timeframe, and vetting during Technical Interchange Meetings. These requirements are listed below.

Spacecraft Vehicle Avionics...

- Shall be capable of functioning in deep space (e.g., beyond LEO)

- Shall be capable of supporting crewed missions

- When uncrewed, shall be capable of autonomous operations for TBD duration

- When uncrewed, shall be capable of being remotely operated from Earth, or elsewhere

- Shall provide capabilities to support science, technology, and research payloads

- Shall support visiting vehicles, both crewed and robotic

- Shall support logistics resupply

- Shall support expansion of vehicle capabilities

- Shall support TBD EVR/EVA proximity operations

- Shall support planetary/surface human/robotic operations

\section{Candidate Scenarios}

In order to define integrated test configurations which could be used to evaluate our capabilities, notional scenarios were developed. These included both crewed and uncrewed operations, as well as a docking between a Multi-Purpose Crew Vehicle (MPCV) and the L2 Station. Although a number of scenarios were considered, only two were used for our final integrated test. The first was a simple MPCV cruise. The second scenario began with an uncrewed L2 Station with MPCV on approach. The MPCV then docked to the L2 Station. Additional crewed operations (e.g., free-flyer operations) were conducted at different phases in this scenario. A detailed description of this scenario is provided in section IV-A.

\section{E. Avionics/Vehicle Functions}

A functional breakdown of vehicle avionics was performed to define needed capabilities for integrated tests, and begin the process of modeling our avionics systems and their simulation support. This functional breakdown included systems functions (e.g., Power Distribution) for each module of the integrated vehicle and Integrated Spacecraft functions (e.g., Integrated Power Control and Distribution) implemented via software. For FY13, these functions were condensed into the Integrated Test \#3 Systems/Objectives shown in Figure 7, but they remain as a framework for modeling and evaluation of future technologies and architectures.

\section{Technical Areas of Emphasis}

Within the AAE project, we are emphasizing the investigation of human interfaces; more powerful processors and network communications; wireless technologies for both networking and instrumentation; and flexible long-haul communications technology. For these areas of emphasis, the high level challenges and architectural guidelines described in section II-C lead to specific technology goals to be addressed during FY13. For example: the challenge of minimizing size, weight, and power drives us to the guideline that wireless technologies be used where possible, and thus the FY13 goal to implement a wireless sensor network and begin to assess its limitations for spaceflight.

\section{A.Human Interfaces}

The goals of AAE Human Interface (HI) work are to identify, adapt, develop and mature innovative, integrated spaceflight human interface technologies that will meet the needs of NASA's planned deep space crewed missions; and to infuse Human Systems Integration (HSI) from beginning to end of the project lifecycle, optimizing the system for crew time, personnel, training, human factors engineering, safety, health, survivability, and cost. Towards these ends, our efforts during FY13 were focused on the development of cockpit displays in F.F using various sensors (including imagery) and effectors located in IPAS.

We also began work on a "Software GPU" to provide GPU functionality using radiation tolerant, safety critical processors suitable for use in a deep space environment. We are now working with the Kennedy Space Center 
(KSC) and, through them, the Center for High-Performance Reconfigurable Computing (CHREC) to determine core requirements of Graphics Processing required by the JSC Human Interfaces team, and to determine low cost, high performance architecture options for future consideration.

In partnership with Honeywell and the Orion program, we also conducted environmental testing on ActiveMatrix Organic Light-Emitting Diode (AMOLED) displays - showing that the AMOLED displays remained functional with some degradation due to Thermal/Vac and radiation testing ${ }^{2}$.

Ultimately, we intend to collect information about command \& telemetry data rates; display view change events; number of displays with live data; data display rates; and cockpit configuration suitability using various display technologies and formats and various control techniques.

In preparation for future exploration missions, we have begun to look at potential Telepresence capabilities with a near-term focus on visual and auditory, and a longer term focus on tactile communication and sensory immersion. We are investigating the impacts of communication latencies and bandwidth variations on the perceived utility of Telepresence.

\section{B. Processors, Networks, and Instrumentation (PNI)}

In this area, our initial areas of investigation center on the use of both time-triggered and non-deterministic Ethernet for all vehicle functions. We are also looking at ways to interface an Ethernet backbone with other control bus architectures. We were able to provide a network configuration for multiple vehicles consisting of sub-nets, wired and wireless. Unfortunately, resource constraints prevented us from fielding a backbone based on timetriggered Ethernet during FY13, but this is an area in which we are making progress during FY14.

We were able to demonstrate the use of computers of different construction, running diverse operating systems to function in a hot swap scenario. In achieving this, the capability and flexibility of Core Flight Software was essential. This approach will allow future flight vehicles a more flexible avionics architecture.

Specifically, we loaded Core Flight Software and verified functionality on a Space Micro Proton400k-L (Dual Core PPC, T-TMR redundancy) using both Linux OS (Version 2.6.37) and VxWorks (Versions 6.8 \& 6.9), and a Maxwell SCS-750 using VxWorks (Version 6.9). We were also able to load the CFS Core Flight Executive on a Raspberry Pi with Broadcom 2835 processor.

During testing, we demonstrated some of the "-ilities" expected to reduce Hardware and Software design Cycle of a spacecraft avionics system, including interchangeability, scalability, flexibility, and maintainability. We were able to demonstrate two dissimilar machines running dissimilar Operating Systems OS's acting in a Hot Spare Configuration.

In order to interface our Ethernet backbone with other bus architectures, we have developed a Common Avionics Enabler (CAE). This device can be configured to handle interfaces with RS-232 and RS-422; interfaces with MILSTD-1553B and Space Wire are in work. During FY13 we were able to develop a Device Discovery Framework for CAE which will permit inter-network Device Discovery during FY14.

We have also utilized a Raspberry Pi processor as a Network Attached Device to monitor network traffic and observe hand controller commands. This was done to show that our network architecture was extensible - the flight bus can be scaled to include networked devices without interference (Consumer of data only) - and to demonstrate the power of small lightweight processor capabilities.

\section{Wireless Sensor Network (WSN)}

We are working to extend terrestrial, standards-based wireless technologies to space applications including sensing, control, and telerobotics. Relevant technologies have been prototyped and evaluated, and infused into the AAE architecture for further study. Our work also supports international space wireless standards development activities and is being coordinated with working groups chartered by the Consultative Committee for Space Data Systems (CCSDS).

We have developed a CFS interface for an ISA100.11a WSN gateway and deployed two small ISA100.11a WSNs on a common vehicle network. We are streaming pressure, temperature, and simulated analog data over ISA100.11a networks through CFS to F.F displays.

We have developed an IEEE 802.11 (Wi-Fi) tele-operated free-flyer analog, shown in Figure 1. This device is remotely controllable over $\mathrm{Wi}-\mathrm{Fi}$, and can stream data and video. 


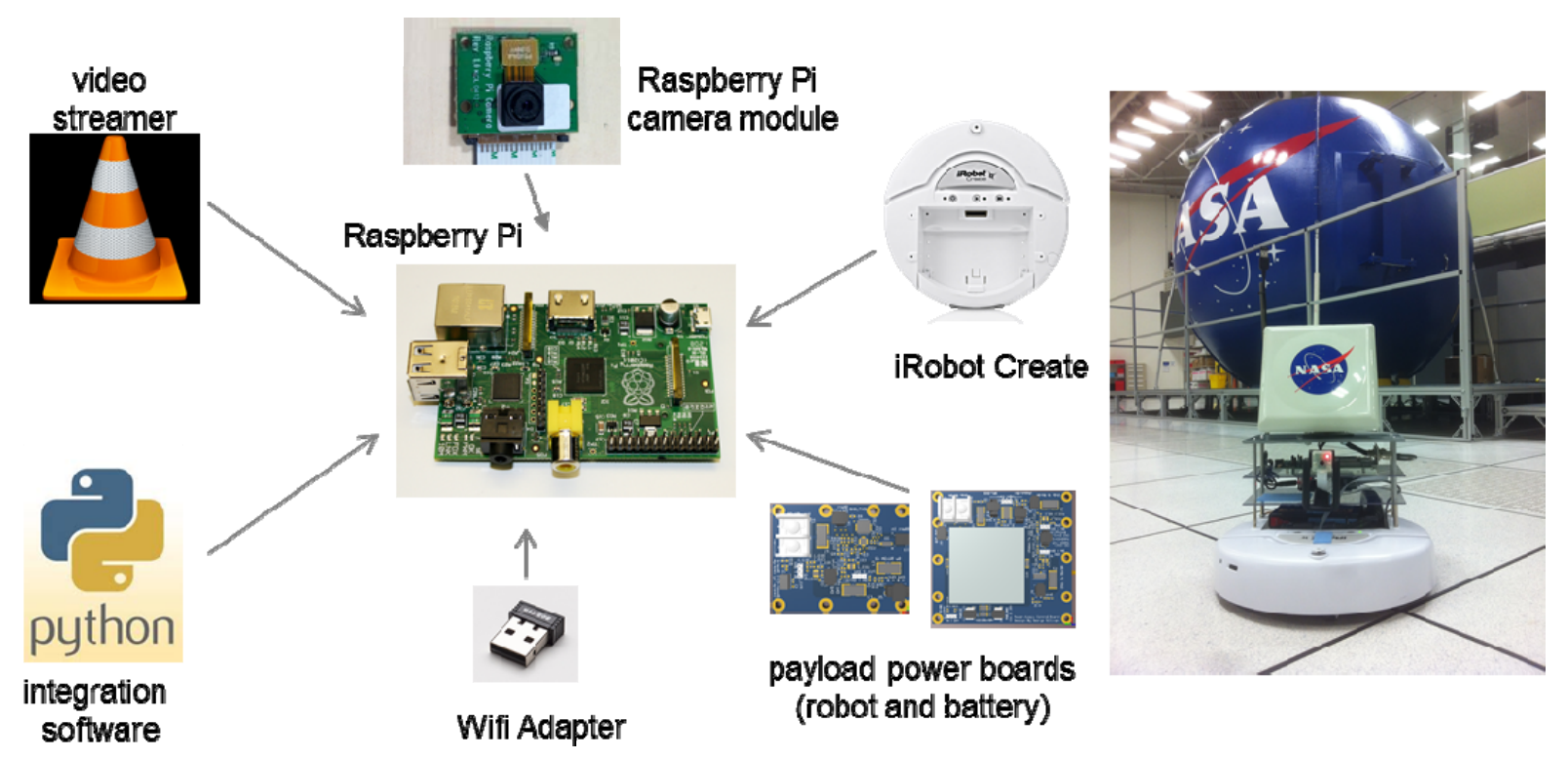

Figure 1. AAE Tele-Operated Free-Flyer

We have used this free-flyer analog to host an RFID interrogator payload and an ultra-wideband (UWB) location, tracking, and sensor data transport payload. Results from this UWB payload are shown in Table 2.

Table 2. Accuracy and precision of 3-Dimensional UWB location estimation

\begin{tabular}{|r|r|r|r|r|r|r|r|r|r|r|r|}
\multicolumn{4}{|c|}{$\begin{array}{c}\text { Target Position } \\
\text { (feet) }\end{array}$} & \multicolumn{3}{c|}{ Tracked Position } & \multicolumn{3}{c|}{$\begin{array}{c}\text { Bias } \\
\text { (feet) }\end{array}$} & \multicolumn{3}{|c|}{ Standard Deviation } \\
\hline $\mathbf{f}$ & $\mathbf{y}$ & $\mathbf{z}$ & $\mathbf{x}$ & $\mathbf{y}$ & $\mathbf{z}$ & $\mathbf{x}$ & $\mathbf{y}$ & $\mathbf{z}$ & $\mathbf{x}$ & $\mathbf{y}$ & $\mathbf{z}$ \\
\hline 0 & 14 & 2.5625 & -0.0452 & 14.0346 & 2.768 & 0.0452 & 0.0346 & 0.2055 & 0.0631 & 0.0663 & 0.1472 \\
\hline-8 & 38 & 2.5625 & -8.0394 & 37.9153 & 2.6532 & 0.0394 & 0.0847 & 0.0907 & 0.0701 & 0.0737 & 0.1951 \\
\hline-16 & 30 & 2.5625 & -16.0383 & 29.9854 & 2.6812 & 0.0383 & 0.0146 & 0.1187 & 0.0697 & 0.0744 & 0.2337 \\
\hline-16 & 12 & 2.5625 & -15.9962 & 12.1353 & 2.9072 & 0.0038 & 0.1353 & 0.3447 & 0.0749 & 0.0793 & 0.1822 \\
\hline-10 & 26 & 2.5625 & -9.993 & 26.0337 & 3.0386 & 0.007 & 0.0337 & 0.4761 & 0.0596 & 0.1002 & 0.2344 \\
\hline
\end{tabular}

In the future we will be collecting a full range of network statistics (e.g., packet latency \& loss rates, throughput, energy consumption, interference tolerance) for a variety of network types, including ISA100.11a, ZigBee, and 802.11n (WiFi).

\section{Communications Architecture and Disruption Tolerant Networking (DTN)}

With the recent advances in microelectronics, wireless transceivers are becoming more versatile, powerful, and portable. This has enabled software-defined radio (SDR) technology, where the radio transceivers perform the baseband processing functions entirely digitally, including modulation/demodulation, error correction coding, and compression/decompression.

To achieve the maximum benefit, an SDR must not only be reconfigurable, but it must also have the ability to observe and measure the state of the operating environment and then intelligently learn and adapt as required. The AAE project is developing these capabilities, which are the fundamentals of cognitive radio (CR) communications.

SDR and CR technologies will be of great benefit to NASA as they allow for increased interoperability due to radio flexibility, interference mitigation by detecting and avoiding potential interferers, higher data throughput by using scarce spectrum efficiently, and lower upgrade costs as the radios are able to adapt to different user/mission needs rather than going through expensive hardware replacement.

During FY13, it was our goal to demonstrate a viable communications architecture for Human Exploration BEO; one that provides core command and telemetry, plus high rate downlink for science, video, and vehicle trending information. 
During FY14, we will be working with our partners at Glenn Research Center (GRC) to port an STRS-compliant platform and libraries into our architecture. This will allow us to understand what it takes to implement STRS compliancy in a COTS SDR.

Because of the cross-agency nature of a communications architecture, we hosted a Technical Interchange Meeting (TIM) with representatives from SCAN, AAE, MOD, and multiple NASA Centers to address Communications for Beyond Earth Orbit Human Exploration. At this TIM we were able to baseline a Communication Architecture for FY13 that included: long haul links from LEO, Near Earth and Deep Space back to Earth, using some variant of a Quadrature Phase Shift Keying (QPSK) and Binary Phase Shift Keying (BPSK); Internal Wireless Sensor Networks using ISA 100.11a; Wireless Local Area Networks using the 802.11 family; and Proximity and surface communications. This communications architecture is diagrammed in Figure 2.

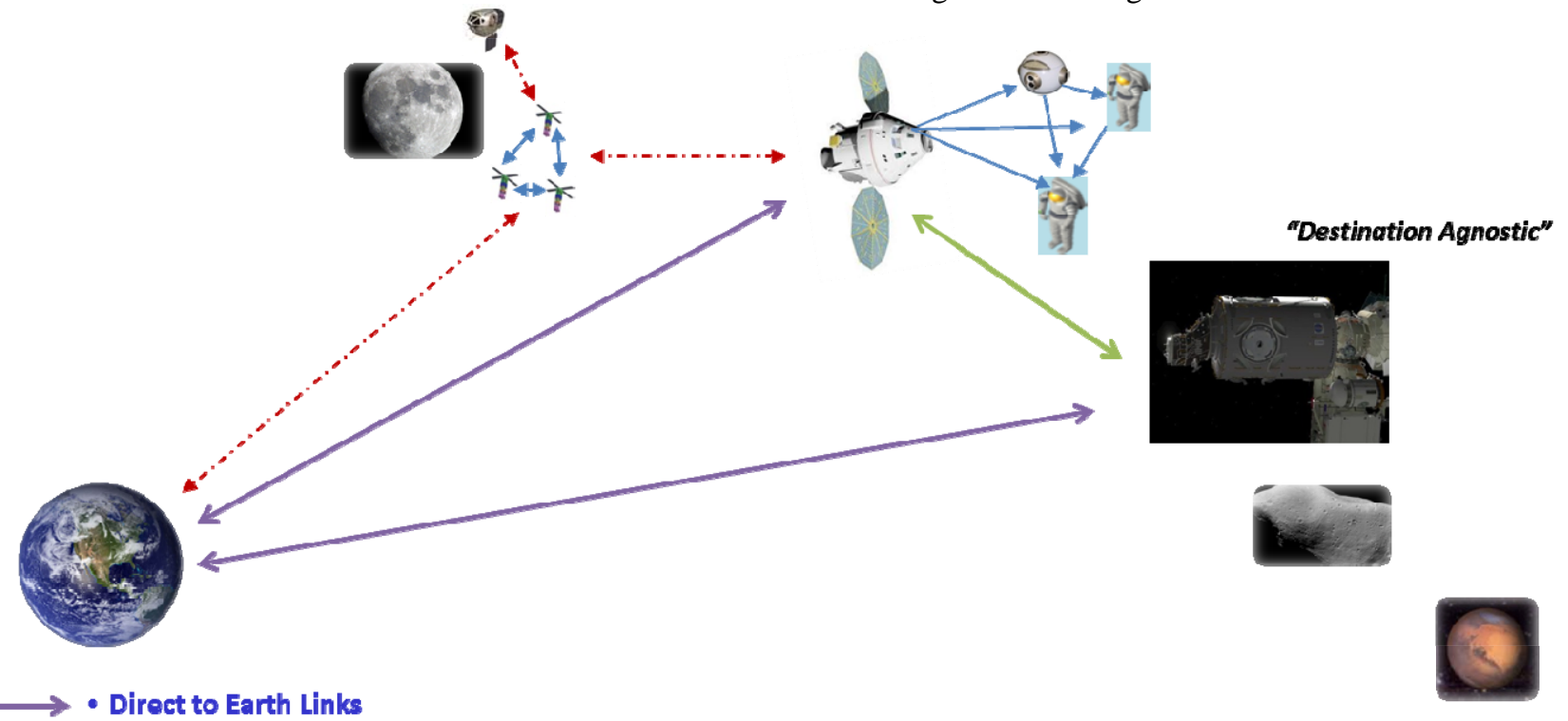

$\longleftrightarrow$ • Direct to Earth Links

- IOAG Service catalog for the mission phase (LEO, Near Earth and Deep Space) - some variant of QPSK and BPSK

$\longleftrightarrow$ - Proximity (vehicle to vehicle) and Surface Communications

- Determine capabilities needed

- look at Proximity-1 and see if that is acceptable or if we need to develop Proximity-2

$\longleftrightarrow$ - Wiroloss local area, mosh notwork -- 802.11 family variant

\& - - • Potential Lunar Relay using Cubesat/Microsat

Figure 2. AAE Baseline Communications Architecture for Human Exploration

Disruption Tolerant Networking (DTN) protocol is a fundamental part of our communications architecture. NASA is developing the DTN protocol suite that extends the terrestrial Internet capabilities into highly stressed data communication environments where the conventional Internet protocols do not work well. The DTN protocol suite is being standardized by the Consultative Committee for Space Data Systems (CCSDS) and all of the DTN protocols will be international standards, supported by open-source software that can help users implement new capabilities.

The development of the DTN protocol is not within the scope of the AAE project, but we are performing trade studies to determine the best location to host DTN within the spacecraft architecture, including key considerations such as size, weight and power, processor utilization, storage capacity, device real estate, and required data rates. The AAE project is also studying the impact of having DTN nodes at other locations within the end-to-end communication architecture, considering factors such as network reliability, implementation costs, and the need for international interoperability.

In addition to baselining the communication architecture, we were able to implement a Space-Vehicle-to-Earth link in our testbed platform, integrate a proximity link with the Space-Vehicle-to-Earth link, and demonstrate endto-end networked data transfer using Free Flyer video going over 802.11 wireless link to on-board vehicle network and over the Space-to-Ground link to the Control Center. 
We completed the pilot engineering program with MathWorks. This effort included the development of a Quadrature Phase Shift Key (QPSK) transceiver system using the MATLAB and Simulink model-based Hardware Description Language (HDL) design tools.

We developed a generic baseband processor software application that can be used with multiple SDR platforms and supports: Framing of CCSDS space packets into Channel Access Data Units (CADUs); Data randomization and Reed-Solomon error correction; multiple data streams (Telemetry, Commands, Voice, Video, etc.); CCSDS formatted packets or generic User Datagram Protocol (UDP); DTN gateway for native or non-native DTN users with integration of ION 3.1.3; Web interface that automatically updates with useful statistics; XML-based configuration files for easy setup and deployment; fill data generation to keep radio link active; and CCSDS packet generation for testing/debugging.

We developed a radio transceiver system using the GNU Radio open-source SDR development architecture targeted for the Universal Software Radio Peripheral (USRP). With this we were able to demonstrate multiple modulation schemes (BPSK, QPSK, GMSK) using a wide range of frequencies on a single platform.

We developed a radio transceiver system using the MATLAB and Simulink model-based Hardware Description Language (HDL) design tools targeted for the Xilinx ML605 Field-Programmable Gate Array (FPGA) development board and the Analog Devices FMCOMMS1-EBZ transceiver daughtercard. When complete, the transceiver system will provide much more bandwidth, and thus higher data rates than the USRP in addition to other added capabilities.

And finally, we were able to procure a low cost Programmable Ultra Lightweight System Adaptable Radio (PULSAR) SDR from MSFC. This radio is expected to arrive in Spring 2014. It supports S-Band Tx/Rx and XBand Tx, BPSK and QPSK, and transmit rates of up to $150 \mathrm{Mbps}$ and receive rates of up to $300 \mathrm{kbps}$

In the future we intend to collect data regarding Baseband processor CPU utilization, FPGA logic utilization for radio/algorithm designs, Throughput rates with/without DTN Bundle Protocol/Licklider Transmission Protocol (BP/LTP), DTN storage requirements for various data types and scenarios, DTN node failure tolerance and recovery times, Packet processing latency and overhead, Packet loss rates and number of DTN retransmissions, and Radio energy consumption

\section{E. Model Based Systems Engineering (MBSE)}

NASA has initiated efforts to implement Model Based System Engineering (MBSE). In an effort to gain experience in this area, the AAE Project adopted this approach for our reference implementations. In the near-term, MBSE tools provide us with the flexibility to analyze and document a variety of architectures and share this information with other AES projects. Ultimately, this will support AAE goal of developing a reference implementation of the avionics architecture(s) that can be provided to industry (users) as a basis for standards and procurements.

During FY13 we selected a Systems Modeling Language (SysML) based tool to support our MBSE implementation, and captured the AAE architecture using it. We initiated development of an AAE SysML component library, completed both a baseline SysML top-level Block Definition Diagram (bdd) of AAE's Physical System Decomposition and Internal Block Diagrams (ibds) for Command \& Data Handling (C\&DH) and Communications (in their as-built configurations).

Figure 3 shows an ibd representation of our vehicle avionics model (circa September 2013), comparable to the Rev 2.0 Architecture shown in Figure 4. 


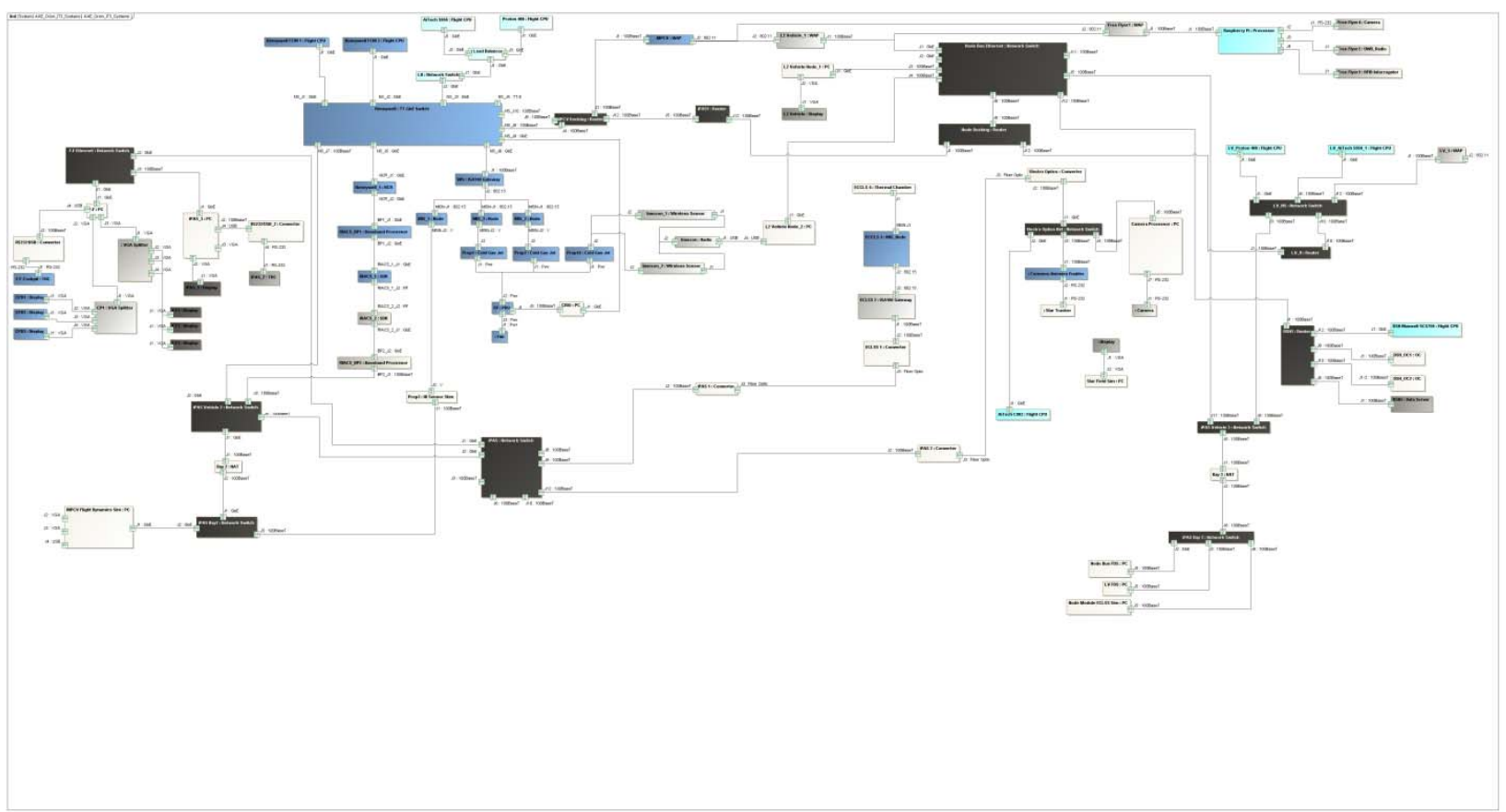

Figure 3. AAE Instantiation using SysML

This model will evolve with design maturity, as more components are added and different architectures are evaluated. Our SysML model will bridge the gap between AAE and iPAS implementation (library vs. instantiation). It will support both flight vehicle analysis and IPAS testing activities. It will enable more automated IPAS reconfiguration and setup, as well as test data mining.

\section{F. Showing a Path-to-Flight}

Our project vision includes the development of architectures and system designs which can be used for flight programs/programs in both the near and long term.

For the near term, this means that we must provide a point solution targeted for an identified mission in 2-3 years. Essentially this solution must be a "good enough" answer from the options we have, which can be matured for flight with minimal effort and used as a basis for procurement specifications.

For the long term, our intent is to build a "catalog" of multiple solutions which can be used "mostly off-theshelf" for a variety of situations. This catalog will include specific components and overall architectures. Recognizing that one size doesn't fit all, each will be rated for suitability to different mission types. New technologies will be incorporated in a timely manner in order to take advantage of strides being made by industry to drive program development and sustaining costs down. This strategy should allow us to include vehicle "hooks \& scars" for later augmentation; facilitate component repair, replacement, and upgrades; and take advantage of schedule slips during the development phase with improved hardware.

Towards these ends, we defined notional processes that included an annual cycle of evaluation to be applied to new architectures/components as they are identified coordinated with a second process which, on request and/or annually, would develop a set of draft products and plans necessary for a selected avionics architecture to achieve PDR level of detail and issue necessary procurements. Gap/risk analyses would be conducted to identify the need for further investigation and maturation of architectures and technologies. This approach balances the Agency needs for continual technological innovation and stabilized technologies for program use.

It was our intent to partially execute these processes during FY13. However, resource constraints combined with our belief that hand-on experience with hardware is of higher priority caused us to put these efforts on hold. 


\section{Integrated Testing}

For the AAE Project, integrated testing provides a "capstone" opportunity for technology developed for each system to be integrated, operated, and tested as part of a candidate architecture during a realistic mission scenario. It also gives us the opportunity to test with larger JSC and NASA communities.

Much of our effort during FY13 was geared around the support of 3 integrated tests distributed throughout the year. Integrated Test \#1, held in March 2013, was used to ensure that we had all of our basic vehicle and test systems in place and to give our personnel experience in the process of testing their capabilities in IPAS. Integrated Test \#2, held in August 2013, was essentially a dry-run for Integrated Test \#3 - allowing us to determine the critical items we needed to work. Integrated Test \#3, held in September 2013, was the culmination of our efforts and was accompanied by a one-day Technology Review of capabilities developed and test results.

\section{A. Mission Scenarios}

Key to the success of IT\#3 was the definition of Mission Scenarios which would fully exercise the capabilities of our architecture and allow us to obtain objective performance data. Two scenarios were used, based on the work described in section II-F, but considerably modified to support test considerations.

The first scenario was simply an "MPCV Cruise", with no significant maneuvers involved. We did this in partnership with the CFS Project to assess the capabilities of the Honeywell 787 FMC running CFS (Ref. Fig. 4, \#1).

The second scenario was considerably more complex, involving MPCV docking with a L2 Station and operations using our free flyer analog and video streaming capabilities. During this scenario, MPCV was emulated using dual dissimilar hardware/OS flight computers running CFS (Ref. Fig. 4, \#2), and a processor failure was introduced in our L2 Station module to demonstrate our "hot swap" capability. A detailed timeline for this scenario is shown in Table 3, together with references to our resultant architecture described in section IV-B, Fig. 4.

Table 3. Scenario 2 Timeline, with Architecture References

\begin{tabular}{|l|l|c|}
\hline \multicolumn{2}{|c|}{ Phase A: Orion Launch to 2000m Separation between MPCV and L2 Station } & Ref \# \\
\hline & $\begin{array}{l}\text { "Pre-Flight": Simulation places MPCV 2000m } \\
\text { from L2 Vehicle and give JSC MCC vehicle control }\end{array}$ & 3 \\
\cline { 2 - 3 } & $\begin{array}{l}\text { KSC LCC has vehicle control and powers up } \\
\text { vehicle, JSC MCC monitors vehicle }\end{array}$ & 3 \\
\hline
\end{tabular}


Table 3. Scenario 2 Timeline, with Architecture References (cont)

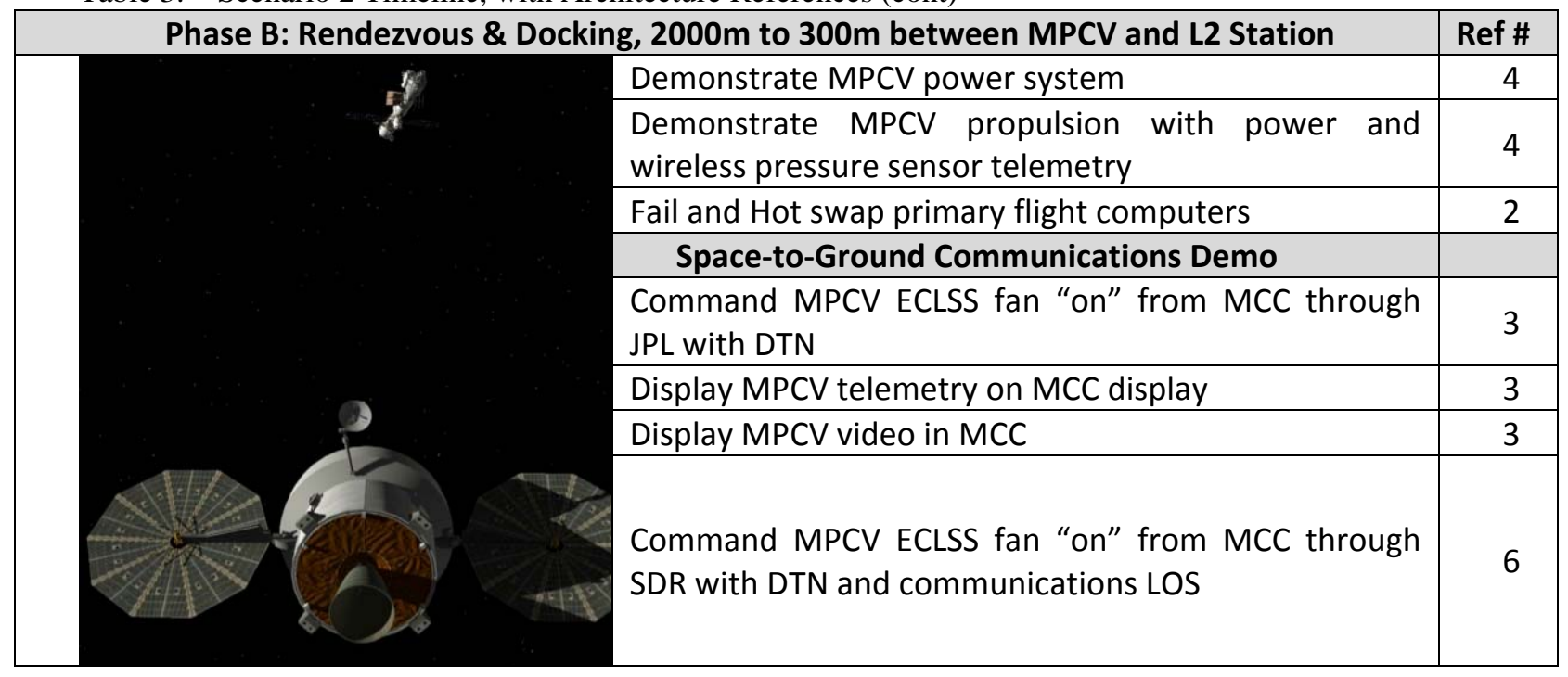

\begin{tabular}{|c|c|c|}
\hline \multicolumn{2}{|c|}{ Phase C: Rendezvous \& Docking, $300 \mathrm{~m}$ to $0 \mathrm{~m}$ between MPCV and L2 Station } & Ref \# \\
\hline \multirow{13}{*}{ N } & Range: $300 m-10 m$ & \multirow[b]{2}{*}{7} \\
\hline & $\begin{array}{l}\text { Observe L2 Vehicle low cabin pressure on MPCV } \\
\text { display through } 802.11 \text { proximity communications } \\
\text { link }\end{array}$ & \\
\hline & $\begin{array}{l}\text { Send Free Flyer from MPCV to L2 Vehicle to } \\
\text { investigate prior to docking }\end{array}$ & \\
\hline & Navigate Free Flyer to L2 Vehicle via 802.11 network & 7 \\
\hline & $\begin{array}{l}\text { Stream video from Free Flyer to MPCV over } 802.11 \\
\text { link and to MCC via SDR }\end{array}$ & 8 \\
\hline & $\begin{array}{l}\text { Demonstrate RFID interrogation of autonomous } \\
\text { sensor node and passive tags }\end{array}$ & 8 \\
\hline & $\begin{array}{l}\text { Demonstrate 3D position tracking with Ultra Wide } \\
\text { Band wireless system }\end{array}$ & 9 \\
\hline & $\begin{array}{l}\text { Demonstrate wireless monitoring of solar panel } \\
\text { perturbations from propulsion jet plumes }\end{array}$ & 10 \\
\hline & Range: $10 m-0 m$ & \\
\hline & Manual docking of MPCV to L2 vehicle & \\
\hline & Use MPCV cockpit to fly vehicle and dock & 9 \\
\hline & Demonstrate MPCV displays & 9 \\
\hline & $\begin{array}{l}\text { Automatically establish wired networking between } \\
\text { vehicles after docking }\end{array}$ & 11 \\
\hline
\end{tabular}




\section{B. Resultant Avionics Architecture}

Based on our FY13 goals, and inputs from our discipline leads and other stakeholders, a high-level architecture was defined for IT\#3. This architecture is shown in Figure 4, along with architecture references tying it back to the Mission Scenario steps described in Table 3. The architecture includes representations of an MPCV, a typical L2 Station module, and a free-flyer analog, all located within IPAS and F.F. It also includes links to locations around JSC and NASA which are used for special purpose emulations of systems, environments, and communications links.

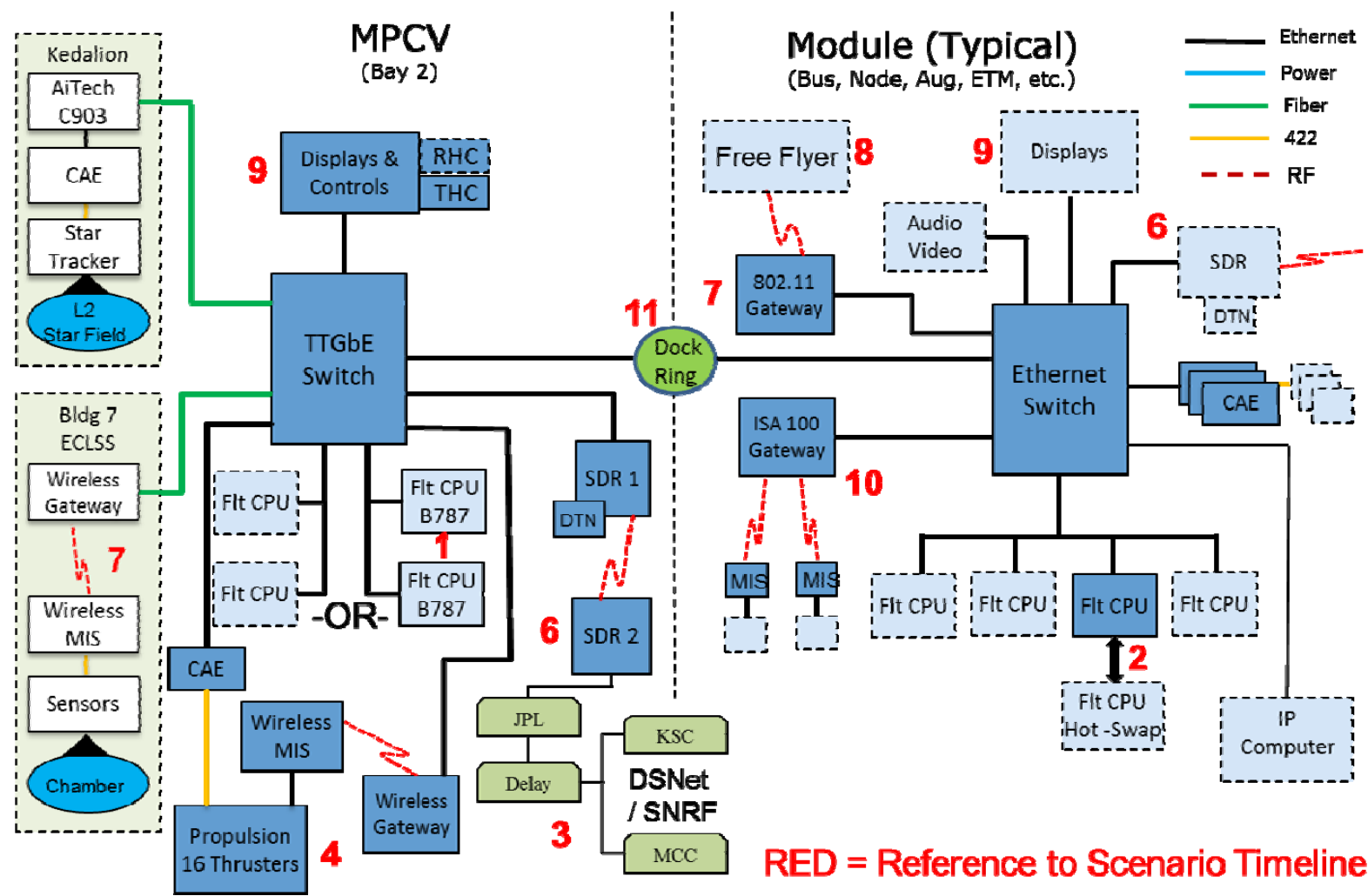

Figure 4. AAE Rev 2.0 Core Architecture, annotated with Mission Scenario steps

\section{C.Integrated Test Results}

Table 4 summarizes our integrated test results, by system and test objectives. Some objectives have been deferred into subsequent FYs, generally due to resource availability issues. Objectives tied to our AMOLEDS evaluation and Software GPU developments were not allocated to a specific Integrated Test, but are also shown in Table 4. 
Table 4. Summary of AAE Integrated Test Results for FY13.

\begin{tabular}{|c|c|c|}
\hline System & Objective & Summary Status \\
\hline \multirow[t]{3}{*}{ Vehicle } & Dock MPCV to Waypoint at L2 & Complete. \\
\hline & $\begin{array}{l}\text { Route data between vehicles using wireless } \\
\text { and hardline connections }\end{array}$ & Complete. \\
\hline & $\begin{array}{lccc}\begin{array}{l}\text { Display spacecraft } \\
\text { spacecraft during rendezvous }\end{array} & \text { on } & \text { other } \\
\end{array}$ & Complete. \\
\hline \multirow[t]{9}{*}{ C\&DH } & $\begin{array}{l}\text { Hot swap dissimilar flight computer during } \\
\text { rendezvous }\end{array}$ & \\
\hline & Network load balancer & $\begin{array}{l}\text { Complete. Used SNMP command to switch for } \\
\text { implementation }\end{array}$ \\
\hline & Port mirror/SNMP heartbeat & Deferred to FY14. Resource issue. \\
\hline & Use Maxwell CPU in DSH & Deferred to FY14. Awaiting new chassis. \\
\hline & $\begin{array}{l}\text { Provide local boot capability for all flight } \\
\text { computers }\end{array}$ & Complete. \\
\hline & Network Appliances & \\
\hline & $\begin{array}{l}\text { Use Raspberry Pi appliance as network } \\
\text { monitor }\end{array}$ & Complete. \\
\hline & Use Raspberry Pi to develop network map & Deferred to FY14. Resource issue. \\
\hline & Fly MPCV with Honeywell FMC & $\begin{array}{l}\text { Complete. GN\&C software running with flight } \\
\text { dynamics sim and can fire jets. }\end{array}$ \\
\hline \multirow[t]{4}{*}{ Networks } & Use routers to dock vehicles & Complete. \\
\hline & $\begin{array}{l}\text { Automatically route spacecraft data across } \\
\text { wireless during prox ops and hardline once } \\
\text { docked }\end{array}$ & Complete. \\
\hline & Use TT-E protocol for Honeywell FMCs & Deferred to FY14. Resource issue. \\
\hline & Route DSH data to L2 & $\begin{array}{l}\text { Tested. Not fully implemented due to limited } \\
\text { resources }\end{array}$ \\
\hline \multirow[t]{13}{*}{$\begin{array}{l}\text { Human } \\
\text { Interfaces }\end{array}$} & Displays & \\
\hline & MPCV ECLSS & $\begin{array}{l}\text { Good data from B7 chamber and with ECLSS sim, } \\
\text { still integrating MIS data to display }\end{array}$ \\
\hline & MPCV thrusters (pwr \& MIS) & Lingering issues with display of MIS data \\
\hline & MPCV TRAJ display (improvements) & Complete. \\
\hline & L2 power (DSH/AMPS) & Complete except for crew display \\
\hline & L2 ECLSS (from sim) & Complete. \\
\hline & MPCV out-the-window view & Complete. \\
\hline & MPCV centerline camera view & Complete. \\
\hline & "God's eye" camera view & Complete. \\
\hline & $\begin{array}{l}\text { Manual docking from F.F with displays } \\
\text { and THC }\end{array}$ & Complete. \\
\hline & Complete AMOLED testing & Complete. \\
\hline & Test sGPU & $\begin{array}{l}\text { Complete. Preliminary tests show improvement } \\
\text { when running on C925 vs. C903. }\end{array}$ \\
\hline & Telepresence comparison & Complete. \\
\hline
\end{tabular}


Table 4. Summary of AAE Integrated Test Results for FY13 (cont).

\begin{tabular}{|c|c|c|}
\hline System & Objective & Summary Status \\
\hline \multirow{6}{*}{$\begin{array}{l}\text { Wireless } \\
\text { Systems }\end{array}$} & Complete CFS ISA 100.11a gateway & Complete. \\
\hline & $\begin{array}{l}\text { Expand ISA100.11a network with more } \\
\text { MIS units }\end{array}$ & $\begin{array}{l}\text { Deferred to FY14, will coordinate with other AES } \\
\text { Projects. }\end{array}$ \\
\hline & $\begin{array}{l}\text { Connect B7 ECLSS chamber with MIS } \\
\text { and CFS gateway }\end{array}$ & Complete. \\
\hline & UWB tracking of free flyer & Complete. \\
\hline & $\begin{array}{l}\text { Integrate } 802.11 \text { MIS for high rate sensor } \\
\text { data }\end{array}$ & Deferred to FY14. Resource issue. \\
\hline & Demonstrate interference tolerance & Deferred to FY14. Resource issue. \\
\hline \multirow[t]{3}{*}{$\begin{array}{l}\text { Commun - } \\
\text { ications }\end{array}$} & $\begin{array}{l}\text { Space-to-ground link using new baseband } \\
\text { processor and USRP }\end{array}$ & Complete. \\
\hline & $\begin{array}{l}\text { Integrate new radio with new baseband } \\
\text { processor }\end{array}$ & $\begin{array}{l}\text { Almost complete. Deferred to FY14 due to limited } \\
\text { resources }\end{array}$ \\
\hline & Integrate DTN into baseband processor & Complete. \\
\hline \multirow[t]{4}{*}{ Free Flyer } & Teleoperate from L2/MPCV & Complete. \\
\hline & Stream video to MCC & Complete. \\
\hline & $\begin{array}{l}\text { RFID interrogator for passive sensors and } \\
\text { inventory }\end{array}$ & Complete. \\
\hline & Add wireless actuation capability & Deferred to FY14. Resource issue. \\
\hline \multirow[t]{6}{*}{ Software } & Executable for Proton CPU & Complete. \\
\hline & Use c903 as star tracker computer & Deferred due to lack of resources \\
\hline & MPCV Telemetry app (Magic Sensor) & Complete. \\
\hline & Add CFDP to FC load & $\begin{array}{l}\text { Endian compatibility issue between CCSDS packet } \\
\text { header and CFS. Deferred due to lack of resources }\end{array}$ \\
\hline & AMPS telemetry app & Complete except for crew display. \\
\hline & Fix Star Tracker CFS app & Deferred due to lack of resources \\
\hline \multirow[t]{4}{*}{$\begin{array}{l}\text { Ground } \\
\text { Systems }\end{array}$} & Start MPCV from KSC LCC & Complete. \\
\hline & $\begin{array}{l}\text { Transfer DOLILU file from KSC LCC to } \\
\text { MPCV }\end{array}$ & Deferred. Awaiting CFDP \\
\hline & Add telemetry and displays in MCC OTF & Deferred due to lack of resources \\
\hline & Receive free flyer video & Complete. \\
\hline GN\&C & Improve star tracker video processing & Requires c903 computer. Deferred due to lack. \\
\hline \multirow[t]{7}{*}{ MBSE } & C\&DH models & Ongoing work to improve fidelity of existing models \\
\hline & Network models & Ongoing work to improve fidelity of existing models \\
\hline & Human interface models & Ongoing work to improve fidelity of existing models \\
\hline & Wireless models & Need models for WAP and MIS \\
\hline & Communications models & Need models for BBSP and radio \\
\hline & Free Flyer models & Complete. \\
\hline & System diagram & Complete. \\
\hline
\end{tabular}




\section{Technology Review}

In conjunction with Integrated Test \#3, the AAE project held a Technology Review. This was used as an opportunity to describe to our stakeholders and colleagues the work that had been done, the results of our efforts, and the work we were considering for the future. Comments were solicited and captured, and these have been incorporated into our planning for FY14 and beyond. We plan to continue this approach in future years.

\section{V.Conclusions}

During FY13, the AAE Project was able to successfully demonstrate a plausible avionics architecture for a notional L2 Station. We were also able to make significant strides toward our goal of a flexible avionics architecture that can be used to evaluate future concepts/architectures/components for both our nominal L2 Station and other vehicles.

Our Rev 2.0 architecture provides a common core system that has standard capabilities and interfaces, and contains basic core elements and functionality needed for any spacecraft. It consists of four key capabilities:

- A common avionics package utilizing modern spaceflight avionics architectures and components to reduce development time and maximize sub-element commonality and reuse.

- A prototype common inter-networked radio system for all future spacecraft, suits, and ground stations, allowing the replacement of the unique capabilities now required for each link with a single type of system throughout the HSF architecture, and resulting in improved operational efficiencies, reduced power, and mass savings.

- Human Interface enhancements that are a mix of commercial and custom (where needed) components that are as common as possible across the AES projects.

- Standardized model-based systems engineering and automated test capabilities to facilitate integration and test.

If desired, this system can be scaled and tailored to any specified mission. The system incorporates hardware from multiple vendors, and reusable and reconfigurable open source software (e.g., GSFC CFS).

Although strides have been made, there remains a great deal of work to do. Our FY14 efforts have been centered around incremental architectural upgrades applied to different mission scenarios. In FY15, we are planning to merge the AAE project with the CFS project, the DTN project, and some aspects of the Autonomous Mission Operations (AMO) project, all funded from AES.

We remain committed to demonstrating the technical and economic benefits of our approach, but the amount of progress we are able to make is dependent on funding and resource constraints, along with the priorities set by the Agency for Human Spaceflight.

Although our team already includes participants from most NASA centers and industry, we recognize the need to widen participation from NASA and other government agencies, add more industry and academic partners, and begin discussions with potential international partners during the coming years. We look forward to engaging these future stakeholders.

\section{Acknowledgments}

The content of this paper represents far more than the efforts of the authors. We would like to thank our industry and academic partners: Honeywell, Draper, Jacobs, Tall Umbrella, and Kansas State University.

The authors would also like to thank all of the members of the AAE Project and IPAS, including:

- From the Langley Research Center (LaRC): Neil Coffey, Satya Kotaru, and Keith Woodman (AAE Deputy Project Manager)

- From the Johnson Space Center (JSC): Chuck Bailey, Rick Barton, Dan Benbeneck, Steve Duran, David Fletcher, Jackie Gillman, David S. Hafermalz, Greg Hall, Chatwin Lansdowne, Mary McCabe, Jacque Myrann, Helen Neighbors, Phong Ngo, David Ni, Bill Othon, Roderick Robinson, Christine Sauers, Adam Schlesinger, Victor Tran, Luis Vazquez, Ray Wagner, Lore Williams (CFS Project Manager)

- From the Kennedy Space Center (KSC): Dave Guibeau

- From the Goddard Space Flight Center (GSFC): Glenn Rakow

- From the Glenn Research Center (GRC): Patrick George, Linda Moore

- From the Jet Propulsion Laboratory (JPL): Dwight Geer

- From the Marshall Space Flight Center (MSFC): Shawn Wallace 
We would also like to offer a special acknowledgment to our sponsors at NASA Headquarters:Jason Crusan (Director, Advanced Exploration Systems Division), and Richard McGinnis (Lead, AES Operations Domain)

\section{References}

${ }^{1}$ W. Othon, "Integrated Power Avionics and Software Environment (iPAS): AES Flight System Technology Maturation for Human Spaceflight”, AIAA Space Ops 2014 (Submitted for Publication)

${ }^{2}$ G. Salazar, "Active Matrix Organic Light Emitting Diode (AMOLED) Environmental Test Report”, JSC66638, November 2013 